\title{
Author Index to Volume 32
}

Aalto TK, 654

Aasen AO, 431

Adderson EE, 257

Advani R, 165

Allen JL, 356, 589

Alpert SE, 87

Alvarez J, 403

Alvaro R, 403

Alvaro RE, 226

Anderson DC, 465

Ando M, 386, 669

Andreoli SP, 360

Andrews D, 165

Anegawa NJ, 483

Angus PW, 39

Anwar M, 542

Arbel E, 530

Aricheta R, 710

Arlazzoroff A, 530

Armstead WM, 114

Arvilommi H, 141

Arvola T, 301

Ascuitto RJ, 236

Assouline JG, 699

Atlas AB, 424

Audí L, 73, 571

Aujard Y, 81

Azaz Y, 417

Bair DK, 585

Ballabriga A, 571

Ballard PL, 673

Ballard RA, 673

Barak Y, 282

Batshaw ML, 483

Baum M, 710

Benlabed M, 412

Benson DW, 10

Benson JD, 683

Berger HM, 399

Berry SA, 169, 600

Bier DM, 455, 693

Birmaher B, 64

Björkander J, 150

Black DD, 553

Blanchette V, 520

Blaner WS, 195

Blau N, 726

Blot P, 81

Bochsler PN, 509

Böhler T, 92

Bonsall I, 175

Bortolussi R, 460

Bos AP, 394

Bos JAH, 595

Bourillon A, 81

Bracken M, 673

Braillon PM, 77

Brandtzaeg P, 145

Brookens MA, 424

Brucklacher RM, 547

Brunet J, 77

Buø L, 431

Buroker CA, 436

Burstein Y, 155

Butler T, 689

Buts J-P, 574

Butte NF, 514

Byatt JC, 296

Cairo MS, 277

Calvelli T, 155
Campbell KA, 333

Carlo WA, 679

Carlsson B, 150

Carrascosa A, 73, 571

Carroll WL, 257

Carr R, 505

Carter AM, 441

Casaer P, 212

Cashore WJ, 524

Cates D, 403

Cates DB, 226

Chao J, 649

Chapman V, 128

Chen F, 230

Chin C, 390

Choy M, 390

Ciletti N, 714

Clahsen PC, 399

Clark EB, 10, 333

Cole FS, 693

Collier RJ, 296

Collins JE, 39

Comens-Keller PG, 296

Cope M, 532

Coquet M, 17

Corey M, 179

Cotton RGH, 726

Couper RTL, 179

Coward WA, 45

Creasy RK, 673

Cuatrecasas JM, 73

Curzi-Dascalova L, 412

Dahl RE, 64

Dalinghaus M, 27

Dancis J, 195

Dandifrosse G, 58

Daniels H, 212

Danko I, 128

Darlow BA, 189

Davies JM, 505

Davies P, 424

Dawson BV, 23

de la Torre SRA, 317

Delmas PD, 77

del Pizzo R, 118

Delpy DT, 532

Dempsher DP, 455

Dennery PA, 87

Deoras KS, 589

D’Ercole AJ, 291

Detmer A, 441

Devlieger H, 212, 605

de Vries L, 212

Dewit $\mathrm{O}, 45$

de Zegher F, 69, 212, 605

DiFiore JM, 679

Dintzis RZ, 376

Dipp S, 649

Dixon RM, 39

Djali S, 483

Dolamore BA, 189

Dolenga M, 479

Duffy DM, 350

Durie PR, 179

Edwards AD, 532

Eggermont E, 212

El-Dahr SS, 649

Eliopoulos C, 520

Eljaafari A, 81

Ellinas $\mathrm{H}, 553$
Elwell CE, 532

Epstein LB, 269

Escourrou P, 412

Evered DF, 472

Ewing CK, 350

Fan J, 714

Ferrández MA, 571

Ferriero DM, 489

Fewell JE, 222

Fisher DJ, 664

Fisher LJ, 179

Fjeld CR, 693

Fleming PJ, 417

Flozak AS, 436

Forbes GB, 502

Forget $\mathrm{P}, 58$

Forstner GG, 179

Fowlkes J, 200

Foz M, 73

Frank L, 215, 407

Freemark M, 200

Friedman WF, 230

Friel JK, 183

Frost Jr JD, 514

Fulginiti VA, 373

Futterweit S, 118

Fyfe JC, 719

Ganapathy V, 125

Gandour-Edwards R, 390

Garlick PJ, 447

Garofalo R, 160

Gaultier C, 412

Gaymes CH, 658

Gee A, 277

George PM, 189

Gerding AM, 27

Gest AL, 585

Gidding SS, 10

Giger U, 719

Gillette PC, 658

Ginns EI, 494

Gittenberger-de Groot AC, 338

Glaze DG, 514

Glorieux FH, 77

Glueck CJ, 559

Goddard-Finegold J, 103

Goldberg M, 530

Goldberg SJ, 23

Golodner M, 155

Gommers D, 595

Goodman DS, 195

Gorry E, 483

Granada ML, 73

Gratama JWC, 27

Greenberg M, 520

Greenspan JS, 356, 589

Greter J, 731

Gross I, 673

Gueron $\mathrm{AB}, 600$

Hahn T, 282

Hahn-Zoric M, 150

Hamza J, 412

Hansen TWR, 524

Hanson LÅ, 150

Hasan SU, 342

Haskins ME, 719

Hatch WC, 155

Hausman GJ, 204

Hechtman P, 479

Heitlinger LA, 312
Heizmann CW, 726

Hellsten E, 645

Hemalatha SG, 169

Henning SJ, 306

Herpe-Patsouris L, 704

Hewett KW, 658

Heyes MP, 483

Hoekelman RA, 499

Hoffman GF, 726

Holani R, 658

Holme E, 731

Holtzer CAJ, 399

Holzknecht RA, 600

Hoyme HE, 23

$\mathrm{Hu}$ N, 333

Hughes AM, 312

Hughes $\mathrm{S}, 10$

Huisman TWA, 338

Huizinga TWJ, 505

Hunte B, 269

Hwang P, 520

Hyun W, 269

Innis SM, 683

Ishizawa L, 277

Isolauri E, 301, 141

Ito T, 386, 669

Jacobson C-E, 731

Jakobs C, 566

Järvelä I, 645

Jennings MG, 296

Jensen CL, 514

Jeusette F, 58

Johansen HT, 431

Johnson P, 417

Johnson PD, 23

Johnston JM, 257

Kabir I, 689

Kadowitz PJ, 236

Kaila M, 141

Kalderon B, 39

Karanth S, 114

Karov Y, 282

Katz J, 195

Kehrer JP, 366

Keklikian EN, 589

Keller BB, 333

Kerr DS, 169

Kim S-B, 436

Kimpen JLL, 160

Kinter MT, 366

Kishimoto TK, 465

Kitamura MY, 132

Kleijer M, 505

Klein N, 520

Klitzner TS, 230

Kneer J, 566

Koers JH, 27

Kohelet D, 530

Kok RM, 566

Konduri GG, 222

Koren G, 520

Korenke GC, 726

Koster JF, 394

Kraak-Slee R, 394

Kraemer LG, 175

Kraemer R, 175

Kramer CM, 87

Kuipers JRG, 27

Kunjummen RD, 465

Kuo S-M, 424 
Kwiatkowski K, 226, 403

L'Abbé MR, 183

Lachmann B, 595

Laine $S, 141$

Larsson A, 595

Larsson N-G, 731

Law P, 277

Lazo JS, 424

Lee PA, 64

Leffler CW, 114

Le Gac S, 81

Leibach FH, 125

Lelievre-Pegorier M, 704

Leo A, 92

Leonard JV, 39

Leroy B, 704

Letellier T, 17

Levin MJ, 699

Levine AD, 269

Levine M, 417

Levitsky LL, 714

Levitz M, 195

Li B UK, 312

Lichtenstein P, 559

Liechti-Gallati S, 175

Lifshitz F, 50

Linderkamp O, 92, 97

Lindstedt S, 731

Liu T, 169

Løberg EM, 107

Locke RG, 356

Lucas A, 73

Lucas AH, 132

Lueder FL, 436

Lyman WD, 155

Machida A, 264

Mahesh VB, 125

Maines MD, 324

Malgat M, 17

Malik N, 175

Mallett C, 360

Mark JA, 324

Martin RJ, 679

Marttinen A, 301

Massa G, 69

Matsui A, 264

Mayock DE, 580

Mazat J-P, 17

McAteer JA, 360

McCabe R, 417

McClung HJ, 312

McCormick DC, 532

McDonald MC, 306

McDonough KH, 236

Medina JM, 317

Mellander L, 150

Mellits ED, 483

Mentzer W, 165

Merlet-Benichou C, 704

Meunier PJ, 77

Meuzelaar JJ, 27

Michael LH, 103

Miller JD, 286

Miller MJ, 679

Mink K, 714

Mirro R, 114

Mishiro S, 264

Mitton SG, 447

Miyazaki H, 264
Moen A, 107

Mogridge N, 189

Moisen RMW, 399

Molenaar JC, 394

Momma K, 386, 669

Momper R, 137

Moon JK, 514

Moore DJ, 179

Moreau E, 704

Moretto B, 17

Moser H, 175

Moya FR, 673

Moyse J, 50

Mujsce DJ, 547

Murphy M, 269

Murray RD, 312

Muskiet FAJ, 27

Nakamoto T, 330

Neilsen NR, 509

Nilsson KO, 731

Noh CI, 658

Northington FJ, 286

Nozaki Y, 264

Nuutinen M, 243

Oberhaensli RD, 39

O'Dorisio TM, 312

Ogata ES, 436

Ogra PL, 160

Oh W, 524

Okamoto H, 264

Oldfors A, 731

Olivieri N, 520

Oltjen S, 390

Orhant E, 412

Osterhaus ADME, 150

Oury JF, 81

$\varnothing$ yasæter S, 107

Padbury J, 673

Parrot-Roulaud F, 17

Partridge JC, 489

Patel MS, 169

Patterson DF, 719

Peebles DM, 532

Peltonen L, 645

Perel J, 64

Phillips S, 664

Piantedosi R, 195

Pierrat V, 212

Pirenne H, 81

Pitt BR, 424

Plunkett JM, 277

Polk DH, 673

Poll-The BT, 566

Ponder DL, 683

Prentice A, 45

Price WA, 291

Radda GK, 39

Rahman MM, 689

Raivio KO, 654

Rajagopalan B, 39

Rajaraman K, 460

Ramage D, 236

Ramamoorthy S, 125

Rantala I, 301

Rashbaum WK, 155

Reynolds EOR, 532

Rigatto H, 226, 403

Rigaux A, 342
Roberts JM, 350

Roberts RJ, 366

Robieux I, 520

Robinson MB, 483

Rocchini AP, 10

Rodriguez-Pierce M, 407

Rognum TO, 145

Rogol AD, 286

Røise O, 431

Romain N, 58

Rong L, 291

Rootwelt T, 107

Rosenberg AA, 537

Ross-Ascuitto NT, 236

Rossowska MJ, 330

Roth SC, 532

Ruedeberg A, 175

Ryan ND, 64

Sakarcan A, 710

Salinas I, 73

Salle BL, 77

Sanmartí A, 73

Santavuori P, 645

Saudubray J-M, 566

Saugstad OD, 107, 431

Scalzo FM, 33

Schärer K, 137

Schneider V, 175

Schor DSM, 566

Schrier S, 165

Schweer H, 137

Serrano MA, 317

Serushago B, 460

Seyberth HW, 137

Shackelford PG, 257

Shaffer TH, 356, 589

Shapiro LJ, 641

Sheldon RA, 489

Sherer DM, 494

Shibata M, 114

Shimamoto GT, 296

Sidransky E, 494

Siegel SF, 64

Siegman JS, 683

Siff TE, 132

Silveri F, 394

Slauson DO, 509

Sluis KB, 189

Sluiter W, 394

Smith JB, 465

Smooker PM, 726

Soeiro R, 155

Sokal EM, 574

Somekh E, 699

Soppi E, 141

Sosenko IRS, 407

Speirs J, 559

Sperl W, 726

Spitz B, 212

Spitz DR, 366

Stadler A, 92

Stadler AA, 97

Standaert TA, 580

Steinberg SA, 719

Stellaard F, 566

Sterkers G, 81

Steve AR, 424

Stewart A, 417

Stiles AD, 291
Stoltenberg L, 145

Straus SE, 699

Tate CA, 664

Tedder DG, 699

Teichberg S, 50

Tenbrinck R, 394

ten Brink HJ, 566

Thomas CR, 441

Thompson K, 455

Thrane PS, 145

Tibboel D, 394

Timms BJM, 679

Tollefsen SE, 455

Tönshoff B, 137

Trachtman H, 118

Tracy T, 559

Trujillo KH, 132

Tsai MY, 600

Tsuda F, 264

Tuchman M, 600

Tulinius M, 731

Turtinen J, 243

Uhari M, 243

Ulreich JB, 23

Underwood LE, 689

Vadgama JV, 472

Vafai A, 699

Vanderschueren-Lodeweyckx M, 69

Vander Straten MC, 585

van de Ven C, 277

Van Hoof F, 574

Vannucci RC, 547

Van Winkle TJ, 719

Vege A, 145

Veldhuis JD, 286, 605

Vesa J, 645

Vilstrup C, 595

Virtanen E, 141

Walenga RW, 87

Wang P, 719

Wapnir RA, 50

Weaver LT, 45

Weintraub Z, 226

Weiss HR, 542

Weiss J, 542

Welliver RC, 160

Werner O, 595

Wetzel GT, 230

Wexler ID, 169

Wilcox CL, 699

Williams D, 277

Williams LV, 360

Williamson DE, 64

Wilson D, 195

Winterbourn CC, 189

Wladimiroff JW, 338

Wolff JA, 128

Wolfson MR, 356, 589

Woodrum DE, 580

Wright NM, 286

Wyatt JS, 532

Yager JY, 547

Yamamoto K, 264

Yano K, 264

Zadik Z, 282

Zheng Q, 714

Zilow EP, 97 